\title{
Estabilidad rotacional y resultados visuales con implante de Iente intraocular enVista TORIC MX60T
}

\section{Rotational stability and visual outcomes with the implantation of the enVista MX60T TORIC intraocular lens}

\author{
Flor D. Guzmán-Iturbe*, Eduardo Chávez-Mondragón, Diego Zamora de la Cruz, \\ Amanda Cáceres-Marín, Daniela Pulido-London y Karla Ruiz-Álvarez \\ Departamento del Segmento Anterior, Instituto de Oftalmología Fundación Conde de Valenciana, Ciudad de México, México
}

\begin{abstract}
Resumen
Objetivo: Evaluar los resultados visuales y la estabilidad rotacional del implante del lente intraocular enVista TORIC MX60T. Metodología: Mediante un estudio prospectivo y longitudinal se realizó cirugía de facoemulsificación con implante del lente enVista TORIC MX60T. A la semana uno, al mes uno y al mes tres se realizó evaluación de la agudeza visual sin corrección (AVSC) y equivalente esférico. Se evaluó en cada cita la alineación del lente tórico mediante Toric Summary del topógrafo OPD-Scan III (Refractive Power/Corneal Analyzer, NIDEK ${ }^{\oplus}$ ). Resultados: Se incluyeron 20 ojos, con una edad promedio de 64.3 años, la media de la longitud axial fue de $23.50 \mathrm{~mm}$. La AVSC preoperatoria promedio fue de $0.84 \log M A R$ $y$ en la visita final fue de $0.004 \log M A R(p<0.0001)$. El astigmatismo corneal preoperatorio promedio fue de -2.50 dioptrías, en la visita final el astigmatismo refractivo fue de -0.34 dioptrías $(p<0.0001)$. La rotación promedio del lente fue de $2.05^{\circ}$, con un rango de $0-5$. No existió relación entre la longitud axial y la cantidad de rotación del lente $(p<0.40)$. La AVSC final se relacionó con el grado de astigmatismo final ( $p=0.001$ ). Ningún paciente requirió capsulotomía posterior y no se observó mediante evaluación clínica desarrollo de glistening. Conclusiones: El lente intraocular enVista TORIC MX60T mostró buenos resultados visuales y excelente estabilidad rotacional a mediano plazo de seguimiento (3 meses), por lo que se puede considerar como una excelente opción terapéutica en pacientes con catarata y astigmatismo corneal regular.
\end{abstract}

Palabras clave: Catarata. Tórico. Astigmatismo. Lente intraocular. EnVista.

\begin{abstract}
Objective: To evaluate the visual results and rotational stability of the enVista MX60T TORIC intraocular lens. Methods: A prospective and longitudinal study was carried out, phacoemulsification surgery was performed with the MX60T toric lens implant. At week one, month one and month three, the uncorrected visual acuity (UVA) and spherical equivalent were evaluated. At each visit, the alignment of the toric lens was evaluated using the OPD-Scan III "toric summary" (Refractive Power/Corneal Analyzer, NIDEK $\left.{ }^{\circledR}\right)$. Results: 20 eyes $(n=20)$ were included. The mean preoperative uncorrected visual acuity (UVA) was $0.84 \log M A R$, and at the final visit UVA was $0.004 \log M A R(p<0.0001)$. The mean preoperative corneal astigmatism was -2.50 diopters, at the final visit the mean refractive astigmatism was -0.34 diopters $(p<0.0001) .90 \%$ of
\end{abstract}

Correspondencia:

*Flor Daniela Guzmán-Iturbe

Laguna de las flores, 1084

Col. La Salle

Fecha de recepción: 28-02-2017

Disponible en internet: 14-05-2018

C.P. 25240, Saltillo, Coah., México

Fecha de aceptación: 10-07-2017

Rev Mex Oftalmol. 2018;92(3):129-135

E-mail: danyguzman@live.com

DOI: 10.24875/RMO.M18000021

www.rmo.com.mx

0187-4519/@ 2018 Sociedad Mexicana de Oftalmología. Publicado por Permanyer México. Este es un artículo Open Access bajo la licencia CC BY-NC-ND (http://creativecommons.org/licenses/by-nc-nd/4.0/). 
the eyes had a final vision of 20/25 or better. The average rotation of the lens was $2.05^{\circ}$ with a range of $0-5$. There was no relation between the axial length and the amount of rotation of the lens $(p<0.40)$. The final UVA was related to the degree of final astigmatism ( $p=0.001$ ). None of the eyes showed glistening neither required posterior YAG capsulotomy. Conclusions: The enVista MX60T toric intraocular lens showed good visual results and excellent rotational stability in the medium term (3 months). Therefore, it can be considered as an excellent therapeutic option in patients with cataract and regular corneal astigmatism.

Key words: Cataract. Tóric. Astimgatism. Intraocular lens. EnVista.

\section{Introduccion}

La catarata es la principal causa de ceguera reversible en el mundo ${ }^{1}$. Los avances en la cirugía de catarata han llevado en la actualidad a la búsqueda de la emetropía ${ }^{2}$. La prevalencia del astigmatismo corneal en sujetos con catarata es variable $y$, aunque en general se reporta de 1 dioptría, llega a ser significativo en el 20 a $22 \%$ de la población ${ }^{3-5}$. Cuando tanto catarata como astigmatismo coexisten siempre debe considerarse la opción de corregir ambas durante el mismo proceso quirúrgico. Existen diversas opciones históricamente realizadas para la corrección del astigmatismo en cirugía de catarata ${ }^{6-10}$; sin embrago, desde la aparición de los lentes tóricos existe cada vez mayor preferencia por esta opción, ya que no tiene las complicaciones inherentes a las incisiones corneales; sin embrago, su principal inconveniente sigue siendo su estabilidad, ya que por cada grado de rotación del lente dentro de la bolsa capsular se pierde el $3.3 \%$ del poder cilíndrico correctivo, y así, un tercio del poder se pierde cuando se llega a una rotación de 10 grados $^{11}$. El lente enVista TORIC MX60T es un lente asférico, de acrílico hidrofóbico (parcialmente hidratado), con fenestraciones en la base de sus hápticas, que posee su poder cilíndrico en la cara posterior, con poderes para la corrección astigmática en el plano corneal de 1.25, $2.00,2.75,3.50,4.25,5.00$ y 5.75 dioptrías (Fig. 1).

\section{Objetivo}

El presente estudio tiene como propósito evaluar los resultados visuales y la estabilidad rotacional en cirugía de catarata con implante del lente enVista TORIC MX60T (Bausch and Lomb ${ }^{\circledR}$ )

\section{Métodos}

\section{Evaluación preoperatoria}

Se realizó un estudio prospectivo, intervencional y longitudinal, basado en la búsqueda de pacientes

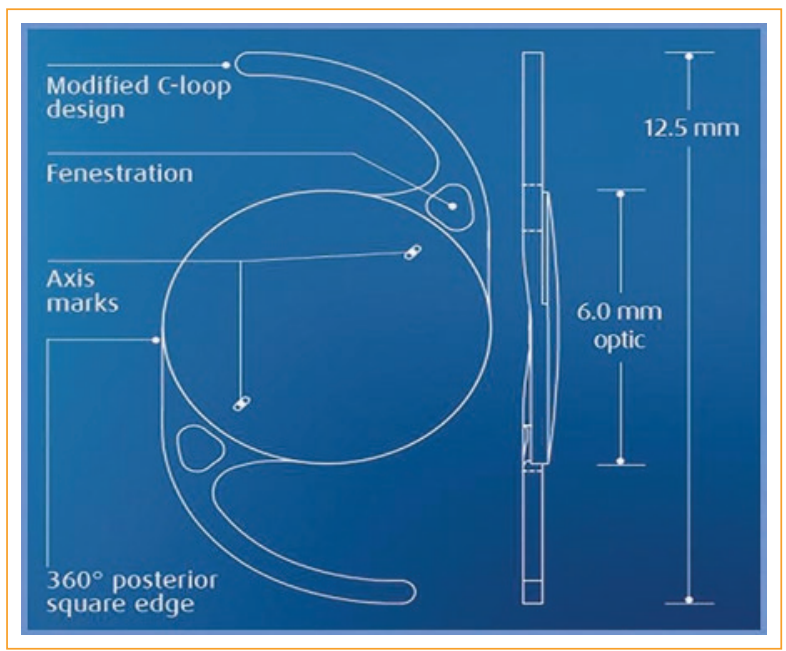

Figura 1. Diseño del lente MX60T.

dentro del Instituto de Oftalmología Fundación Conde de Valenciana. El estudio cumplió con los criterios de Helsinski y fue aprobado por el comité de investigación y de ética en investigación de la institución. Se incluyeron todos aquellos sujetos con catarata senil y baja visual atribuible a la opacidad del cristalino que además tuvieran un astigmatismo corneal regular de entre 1.00 y 4.50 dioptrías (OPD-Scan III Refractive Power/ Corneal Analyzer, NIDEK ${ }^{\circledR}$ ) (Fig. 2). Se realizó una exploración oftalmológica completa, se excluyeron pacientes con cualquier patología ocular o sistémica que pudiera afectar el resultado visual postoperatorio, como alta miopía, glaucoma, retinopatía diabética o degeneración macular relacionada con la edad, también se excluyeron pacientes con patología ocular que afectara la estabilidad del lente dentro de la bolsa, como diálisis zonular traumática o debilidad zonular secundaria a síndrome de pseudoexfoliación. A todos estos pacientes se les informó sobre el protocolo de investigación, aquellos que aceptaron formar parte del mismo se les entregó un consentimiento informado que firmaron.

El cálculo de la potencia esférica del lente se realizó con la fórmula $S R K / T$, con datos obtenidos de la realización de los siguientes estudios: 


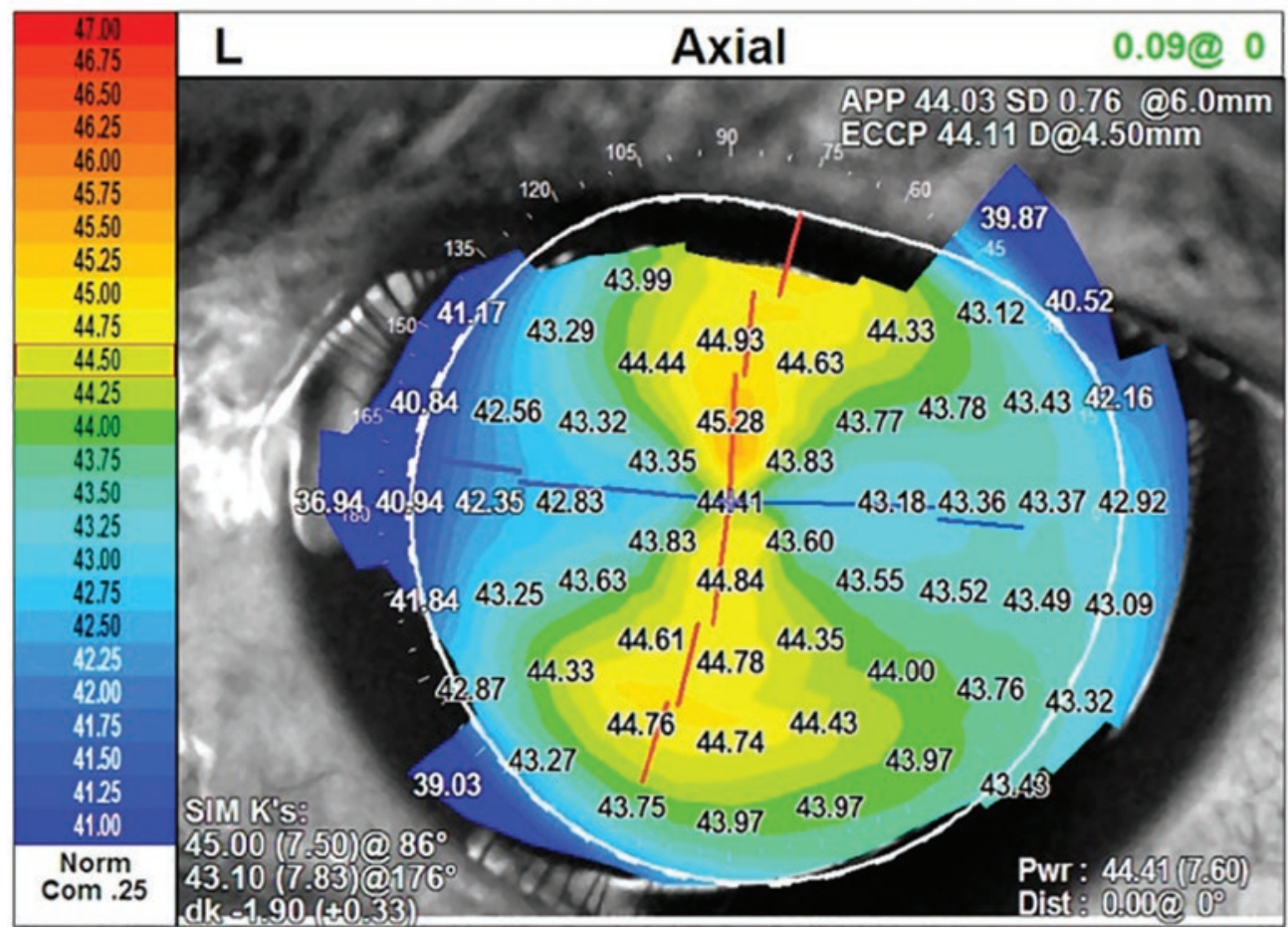

Figura 2. Topografía, mapa axial que muestra astigmatismo corneal simétrico y ortogonal.

- Biometría ocular mediante interferometría (AL-Scan Optical Biometer, NIDEK ${ }^{\circledR}$ )

- Promedio queratométrico basado en topografía corneal (OPD-Scan III Refractive Power/Corneal Analyzer, $\mathrm{NIDEK}^{\circledR}$ )

El cálculo de la alineación del lente tórico se realizó con el software del calculador de Bausch and Lomb ${ }^{\circledR}$, disponible en la página de internet, al trasladar los valores queratométricos obtenidos mediante la topografía corneal tomada previamente. https://envista.toriccalculator.com (Fig. 3).

\section{Técnica quirúrgica}

El día de la cirugía se realizó marcaje en lámpara de hendidura mediante desepitelización corneal minutos antes de la cirugía a 0 y $180^{\circ}$. Se realizó aseo con povidona yodada al $10 \%$ en la superficie de la piel y al $5 \%$ en la superficie conjuntival, y después de la colocación de los campos estériles y antes de la incisión corneal se realizó el marcaje indicado por el calculador tomando como referencia las marcas mencionadas previamente. Posteriormente se realizó cirugía de catarata mediante facoemulsificación con incisión de $2.8 \mathrm{~mm}$ en cornea clara; se utilizó azul de trípano solo en caso necesario, determinado de forma subjetiva por el cirujano; se realizó capsulorrexis circular continua de 4.5-5 mm de diámetro; se facoemulsificó el núcleo con técnica de stop and chop, y se realizó aspiración de restos corticales en forma coaxial. Durante la cirugía se utilizó viscoelástico Amvisc Plus ${ }^{\circledR}$ (hialuronato de sodio al $1.6 \%$, Bausch and $\operatorname{Lomb}^{\circledR}$ ), con posterior implante del lente MX60T, alineación 5-10 grados previos al preestablecido, enfatizando la aspiración del viscoelástico entre el lente y la bolsa capsular para evitar rotación del mismo en el postoperatorio. Cabe destacar que, dentro de la bolsa, el lente puede realizar rotación a favor y en contra de las manecillas del reloj hasta tener la posición final. Los procedimientos fueron realizados por el mismo subespecialista en Microcirugía del Segmento Anterior. En el postoperatorio inmediato se realizó tratamiento tópico con prednisolona al 1\% y moxifloxacino. El esteroide se redujo de forma paulatina de acuerdo al grado de inflamación postoperatoria.

\section{Evaluación postoperatoria}

La revisión fue la habitual, a las 24 horas y a la semana, con visitas adicionales en caso necesario de acuerdo a la evolución clínica individual. Las siguientes 
variables fueron evaluadas a la semana uno, al mes uno y al mes tres: agudeza visual lejana sin corrección (con la tabla de Snellen convertida a logMAR), agudeza visual lejana con corrección (con la tabla de Snellen convertida a logMAR), error refractivo residual expresado en equivalente esférico (EE) y alineación en grados del lente tórico evaluado bajo midriasis farmacológica máxima (Fig. 4) mediante el evaluador OPDScan III Refractive Power/Corneal Analyzer, NIDEK ${ }^{\circledR}$, utilizando la función disponible en el software del programa llamada Toric Summary para medir de forma manual el grado de alineación (Fig. 5). En cada cita se realizó, además, una evaluación mediante lámpara de hendidura de la presencia o ausencia de opacidad de cápsula posterior, así como evaluación de la presencia de glistening.

\section{Análisis estadístico}

Se realizó análisis de los resultados mediante la prueba de Wilcoxon para la comparación de medianas, con un intervalo de confianza del $95 \%$, y se realizaron pruebas de correlación de Pearson para asociación entre variables. La recolección y el análisis de los datos se realizó mediante el programa Microsoft Office Excel 2010 y con el programa IBM SPSS Statistics versión 20. Se consideró un valor $p$ estadísticamente significativo cuando fue menor de 0.05 .

\section{Resultados}

Se incluyeron en el estudio un total de 20 ojos de 16 pacientes $(n=20)$, ningún paciente fue eliminado. La edad promedio fue de $64.3 \pm 9.9$ años, el $95 \%$ fueron mujeres. En cuanto a las características oculares preoperatorias, la lateralidad fue el $60 \%$ de ojos izquierdos y el $40 \%$ de ojos derechos, la longitud axial promedio fue de $23.50 \pm 0.90 \mathrm{~mm}$, el astigmatismo corneal promedio fue de $2.53 \pm 0.98$ dioptrías, con un promedio queratométrico de $43.83 \pm 1.34$. El promedio del poder esférico del lente intraocular implantado fue de 21.25 dioptrías. La agudeza visual sin corrección (AVSC) promedio preoperatoria fue de $0.84 \pm 0.48$ en logMAR, con una mediana de 0.76 , y la agudeza visual mejor corregida (AVMC) fue de $0.585 \pm 0.41$ en logMAR, con una mediana de 0.53 . En la tabla 1 se muestra un resumen de las características preoperatorias.

No existieron complicaciones trans o posquirúrgicas, todos los sujetos completaron el seguimiento. La AVSC promedio postoperatoria final fue de $0.004 \pm 0.19$ logMAR. Al realizar la comparación entre la AVSC inicial

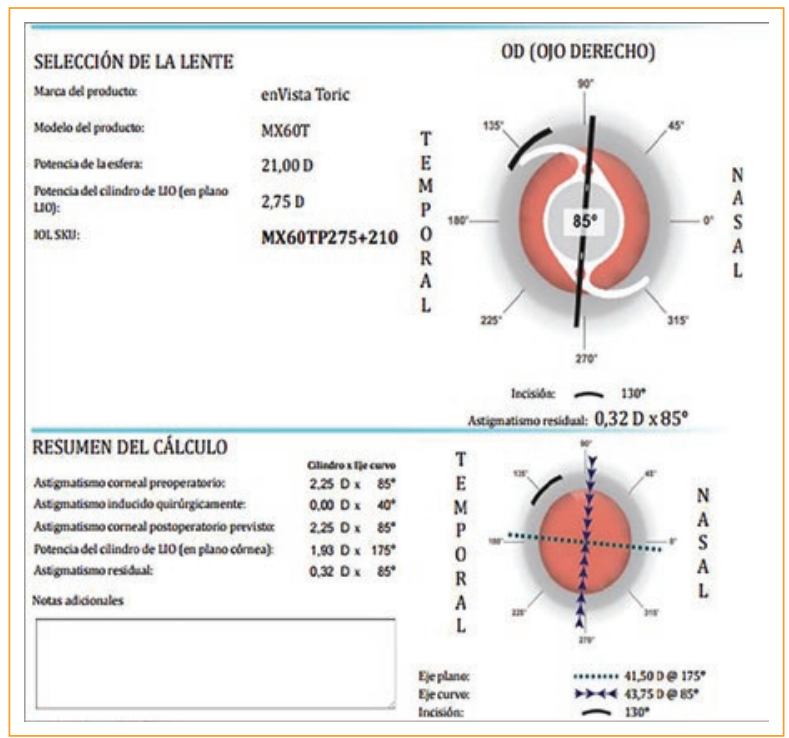

Figura 3. Ejemplo de datos arrojados por el calculador en línea.

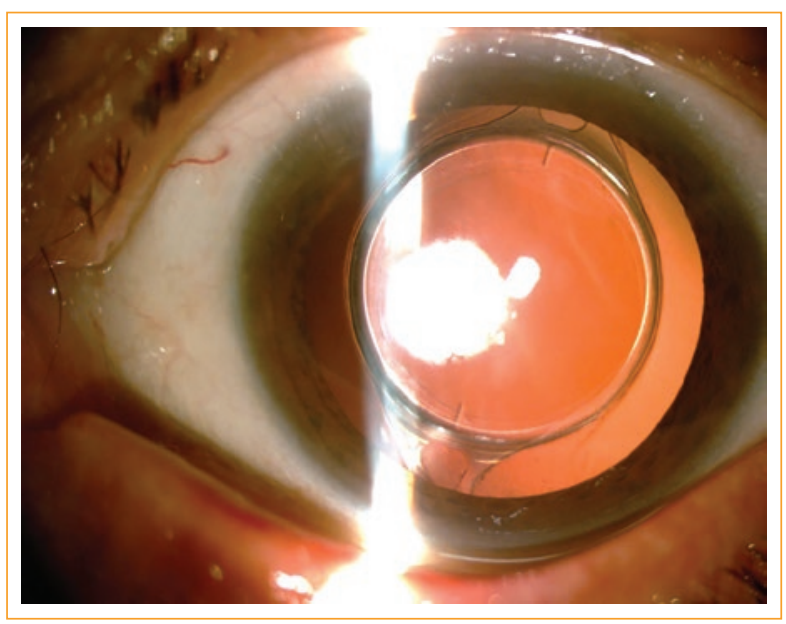

Figura 4. Imagen del lente evaluado bajo midriasis farmacológica.

y final se obtuvo significancia estadística $(p<0.0001)$. El EE promedio postoperatorio fue de $-0.30 \pm 0.23$, en cuanto al astigmatismo refractivo final se encontró un promedio de $0.34 \pm 0.51$ dioptrías, cuando se realizó la comparación entre el astigmatismo corneal preoperatorio y refractivo final se encontró que la diferencia fue estadísticamente significativa $(p<0.0001)$, el astigmatismo corneal promedio postoperatorio fue de 2.37 \pm 1.01 y al realizar la comparación entre el promedio pre y postoperatorio no se encontró significancia estadística ( $p>0.48$ ). El $90 \%$ de los ojos tuvo una AVSC 


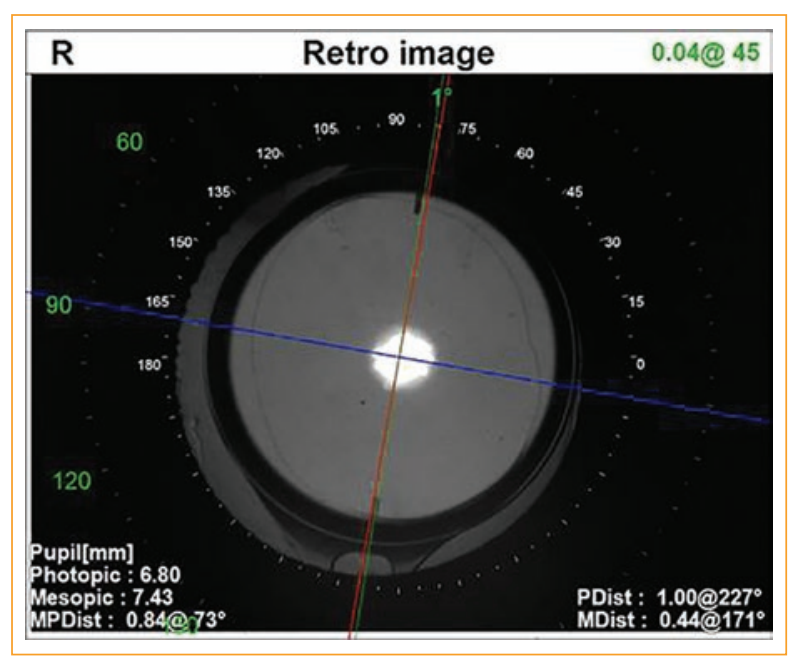

Figura 5. Ejemplo de la imagen obtenida del OPD-Scan III en el que se muestra una línea roja que corresponde al eje más curvo corneal (eje de alineación del lente tórico), una línea azul que corresponde al eje más plano corneal y una línea verde que se pude manipular en forma manual para hacerla corresponder con las marcas del lente tórico colocado, en este caso se observa que hay una pérdida de alineación de $1^{\circ}$ (espacio entre la línea roja y verde).

final igual o mejor a 20/25. La rotación promedio respecto el eje previsto fue de $2.05^{\circ}$ con un rango desde 0 hasta 5 , el $85 \%$ de los lentes tuvieron una rotación menor a $3^{\circ}$ (Tablas 2 y 3 y Fig. 6), al realizar la correlación entre la cantidad de rotación del lente y la longitud axial no se encontró significancia estadística $(p>0.40)$.

Se encontró una relación lineal negativa con significancia estadística $(p=0.001)$ cuando se comparó la AVSC final y el astigmatismo refractivo final ( $p$ de Pearson $=0.690)($ Fig. 7).

\section{Discusión}

La corrección del astigmatismo corneal en cirugía de catarata tiene sus mejores resultados cuando se implanta un lente tórico, donde la estabilidad rotacional es la característica más importante, ya que como se mencionó existe una pérdida del 3.3\% de la corrección total del astigmatismo por cada grado de rotación del lente. Con los excelentes resultados reportados con lentes tóricos, su uso se ha convertido en una indicación y no en una opción en pacientes que son buenos candidatos.
Tabla 1. Características preoperatorias

\begin{tabular}{l|c}
\hline Longitud axial promedio & $23.50 \pm 0.90 \mathrm{~mm}$ \\
\hline Promedio queratométrico & $43.83 \pm 1.34$ \\
\hline Astigmatismo corneal promedio & $-2.50 \pm 0.98$ dioptrías \\
\hline AVSC promedio (logMAR) & $0.841 \pm 0.48$ \\
\hline AVMC promedio (logMAR) & $0.585 \pm 0.41$
\end{tabular}

AVMC: agudeza visual mejor corregida; AVSC: agudeza visual sin corrección.

Tabla 2. Características postoperatorias

\begin{tabular}{|l|c|}
\hline AVSC promedio (logMAR) & $0.004 \pm 0.19$ \\
\hline Astigmatismo refractivo promedio & $-0.34 \pm 0.51$ \\
\hline Equivalente esférico promedio & $-0.30 \pm 0.23$ \\
\hline Rotación promedio & $2.05^{\circ}$ \\
\hline
\end{tabular}

AVSC: agudeza visual sin corrección.

Los resultados obtenidos en el presente estudio son similares, incluso mejores, en comparación con otros estudios realizados en cuanto a estabilidad rotacional de lentes tóricos y resultados visuales. Al hablar de diferentes diseños de lentes nos encontramos que Zarranz, et al. realizaron una evaluación del lente intraocular tórico Acrysof ${ }^{\circledR}$, que tiene una plataforma similar al MX60T con hápticas en $\mathrm{C}$, analizando un total de 54 ojos y un seguimiento a 2 meses. El astigmatismo preoperatorio promedio fue de -2.25 dioptrías y en el seguimiento final fue de -0.32 dioptrías ( $p<0.001$ ); la rotación media durante el seguimiento fue de $3.87^{\circ}$, por lo tanto, fue mayor en comparación a nuestros resultados ${ }^{12}$. Bascaran et al. estudiaron los resultados visuales, así como la estabilidad rotacional del lente tórico AT TORB $\left.\right|^{\circledR} 709 \mathrm{M}$, que, a diferencia del lente utilizado en nuestro protocolo, tiene una plataforma de plato. Se incluyeron 48 ojos y encontraron una AVSC de $20 / 40$ o mejor en el $88.1 \%$ de los casos, con un seguimiento a 6 meses, y mejor de 20/25 en el $61.9 \%$ de los casos. El cilindro promedio disminuyó de -2.24 a -0.43 en el postoperatorio, la rotación promedio en este estudio fue de $4.42^{\circ}$ (desde 0 hasta $16^{\circ}$ ), el $86 \%$ rotaron menos de 10 grados $^{13}$. Lubinski, et al. estudiaron a 6 meses 27 ojos con implante de lente intraocular Tecnis ${ }^{\circledR}$ Toric ZCT (Abbot Medical Optics), que cuenta con plataforma de óptico y hápticas 
Tabla 3. Comparación de variables pareadas (AVSC y astigmatismo)

\begin{tabular}{|l|c|c|c|}
\hline & Preoperatorio & Final & Valor p \\
\hline AVSC promedio (logMAR) & 0.841 & 0.004 & $<0.0001$ \\
\hline Astigmatismo promedio & -2.5 (corneal) & -0.34 (refractivo) & $<0.0001$ \\
& & -2.37 (corneal) & $>0.48$ \\
\hline
\end{tabular}

AVSC: agudeza visual sin corrección.

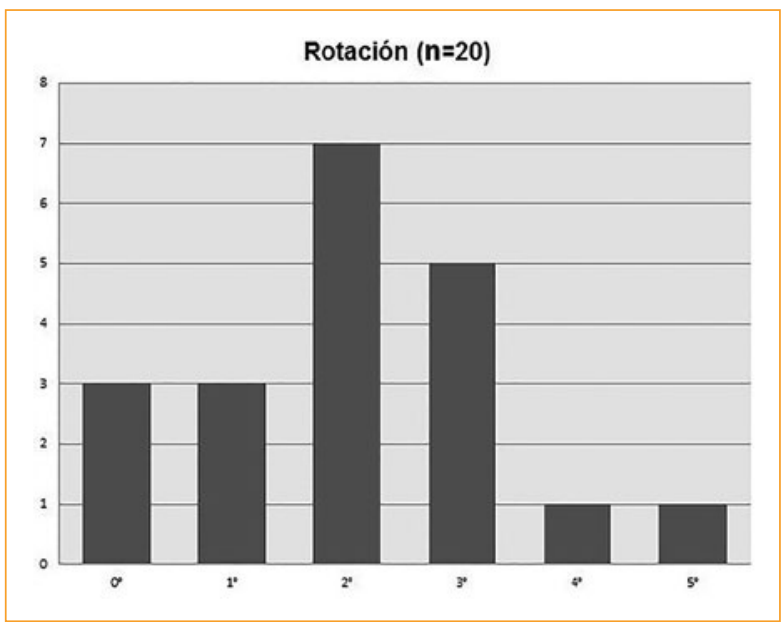

Figura 6. Frecuencia para cada grado de rotación del lente.

en $\mathrm{C}$, similar al Acryosof ${ }^{\circledR}$ y MX60T. En sus resultados tuvieron dos pacientes con rotación mayor a $5^{\circ}$, a los que se les recolocó el lente en una segunda cirugía, la agudeza visual promedio final fue de 20/27 ( 0.14 en logMAR); el cilindro disminuyó de -3.73 a -1.42 dioptrías sin encontrar cambios en el cilindro queratométrico, y la rotación promedio fue de $1.1^{\circ}$, un poco menor a la encontrada en los resultados de nuestro estudio, en donde la agudeza visual promedio mejoró de 0.84 en logMAR a 0.004 , lo que fue estadísticamente significativo, y el astigmatismo promedio cambió de -2.50 a -0.34 ( $p<0.001)$, con una rotación promedio de $2.05^{\circ}$ (rango desde $\left.0-5^{\circ}\right)^{14}$. Sheppard realizó una evaluación del mismo lente, con un seguimiento de 2 meses y encontró una AVSC final de 0.15 (logMAR), con una rotación promedio de $3.4^{015}$. Ferreira et al. realizaron una comparación de los resultados visuales de dos lentes tóricos AMO Tecnis ${ }^{\circledR}$ y Alcon Acrysof $^{\circledR}$, con un total de 40 ojos entre ambos grupos, sin encontrar diferencias significativas estadísticamente al comparar AVSC, AVMC, EE y astigmatismo refractivo residual. El primer grupo con una rotación

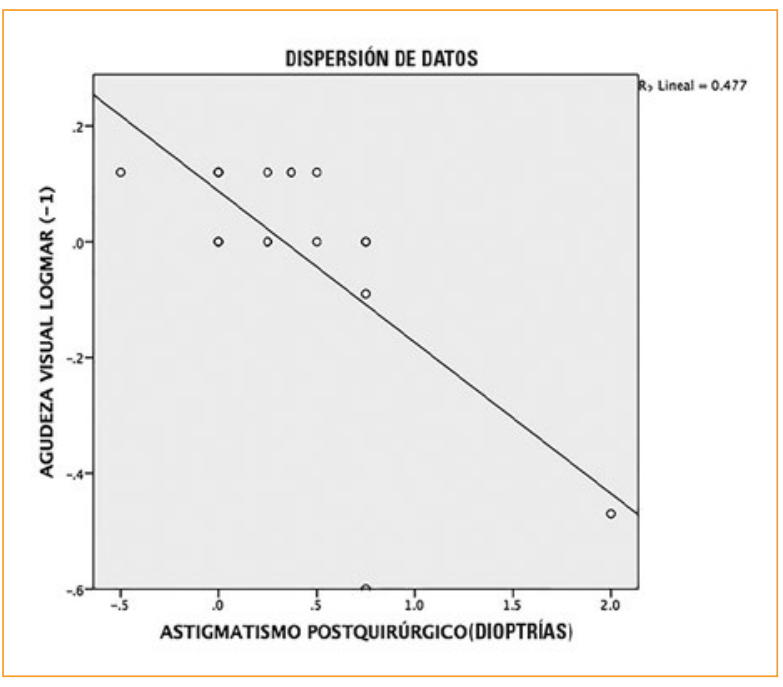

Figura 7. Tabla de dispersión de puntos en donde se observa la relación lineal entre la mayor AVSC final y menor astigmatismo refractivo final.

media de $3.25^{\circ}$ y el segundo de $3.15^{\circ}$. Ningún caso tuvo rotación mayor a $10^{\circ 16}$.

Por supuesto, es difícil que la comparación de los resultados visuales y estabilidad rotacional con otros estudios sea fidedigna, ya que las diferencias en la metodología, incluyendo las características de los pacientes, el tipo de topógrafo utilizado para la obtener las queratometrías, el método utilizado para la biometría óptica (aplanación, inmersión o interferometría), el método utilizado para el marcaje corneal y la técnica quirúrgica, así como el método utilizado para evaluar el eje del lente en el postoperatorio, influyen en los resultados. Por supuesto, siempre se deben tener en cuenta factores ya conocidos que tienen un impacto negativo en la estabilidad rotacional de cualquier lente tórico, como las longitudes axiales mayores a $25 \mathrm{~mm}$ (con una distancia blanco-blanco grande), astigmatismo con la regla, material del lente diferente al acrílico, remoción incompleta de viscoelástico, así como el tamaño de la capsulorrexis cuando esta no cubre el total del óptico, los 360 grados $^{17,18}$. 
Como limitación principal en nuestro estudio se encuentra el tamaño de la muestra y el seguimiento que fue a mediano plazo, ambas posiblemente modificables en el futuro. En nuestro estudio fue fundamental la cantidad de rotación del lente para el resultado visual final, ya que como se reportó, a mayor astigmatismo refractivo final peor agudeza visual final. El caso con peor agudeza visual final fue el que tuvo mayor cantidad de rotación del lente, el cual, además, no mejoró por una probable ambliopía refractiva.

\section{Conclusión}

El lente intraocular enVista ${ }^{\circledR}$ TORIC MX60T mostró buenos resultados visuales y excelente estabilidad rotacional a mediano plazo de seguimiento (3 meses), por lo que se puede considerar como una opción terapéutica en pacientes con catarata y astigmatismo corneal regular.

\section{Responsabilidades éticas}

Protección de personas y animales. Los autores declaran que para esta investigación no se han realizado experimentos en seres humanos ni en animales.

Confidencialidad de los datos. Los autores declaran que han seguido los protocolos de su centro de trabajo sobre la publicación de datos de pacientes.

Derecho a la privacidad y consentimiento informado. Los autores han obtenido el consentimiento informado de los pacientes y/o sujetos referidos en el artículo. Este documento obra en poder del autor de correspondencia.

\section{Financiamiento}

Los autores declaran que los lentes intraoculares utilizados en este protocolo de investigación fueron donados por la empresa Bausch \& Lomb.

\section{Conflicto de intereses}

Los autores no tienen ningún conflicto de interés en este estudio.

\section{Bibliografía}

1. Prokofyeva E, Wegener A, Zrenner E. Cataract prevalence and prevention in Europe: a literature review. Acta Ophthalmol. 2013;91:395-405.

2. Zarranz J, Moreno J, Caire J, González J, Fernández E, Sádaba L. Implante de lentes intraoculares tóricas Acrysof ${ }^{\circledR}$ en cirugía de la catarata. Arch Soc Esp Oftalmol. 2010;85(8):274-7.

3. Hoffer KJ. Biometry of 7500 cataractous eyes. Am J Ophtalmol 1980;90:360-368.

4. Ferrer-Blasco T, Montés-Micó R, Peixoto-de-Matos SC, GonzálezMéijome JM, Cerviño A. Prevalence of corneal astigmatism before cataract surgery. J Cataract Refract Surg. 2009;35:70-5.

5. Khan M, Muhtaseb M. Prevalence of corneal astigmatism in patients having routine cataract surgery at a teaching hospital in the United Kingdom. J Cataract Refract Surg. 2011:37:1751-5.

6. Diakonis V, Yesilirmak N, Cabot F, Kankariya V, Kounis G, Warren D, et al. Comparison of surgically induced astigmatism between femtosecond laser and manual clear corneal incisions for cataract surgery. $\mathrm{J} \mathrm{Ca-}$ taract Refract Surg. 2015;41:2075-80.

7. Kymionis G, Yoo S, Ide T, Culbertson W. Femtosecond-assisted astigmatic keratotomy for post-keratoplasty irregular astigmatism. J Cataract Refract Surg. 2009;35:11-3.

8. Hirnschall N, Gangwani V, Crnej A, Koshy J, Maurino V, Findl O. Correction of moderate corneal astigmatism during cataract surgery: Toric intraocular lens versus peripheral corneal relaxing incisions. J Cataract Refract Surg. 2014;40:354-61.

9. Budak K, Friedman NJ, Koch DD. Limbal relaxing incisions wirh cataract surgery. J Cataract Refract Surg. 1998;24:503-8.

10. Ayala M, Pérez-Santoja J, Artola A, Claramonte P, Alió JL. Laser in situ keratomileusis to correct residual myopia after cataract surgery. J Refract Surg. 2001;17:12-6.

11. Novis C. Astigmatism and toric intraocular lenses. Curr Opin Ophthalmol. 2000;11(1):47-50

12. Zarranz J, Moreno J, González J, Fernández E, Sádaba LM. Acrysof ${ }^{\circledR}$ toric intraocular lens implantation in cataract surgery. Arch Soc Esp Oftalmol. 2010;85(8):274-7.

13. Bascaran L, Mendicute J, Macias B, Arbelaitz N, Martinez I. Efficacy and stability of AT TORBI $709 \mathrm{M}$ toric IOL. J Refract Surg. 2013;29(3):194-9.

14. Lubiński W, Kaźmierczak B, Gronkowska-Serafin J, Podbor『czy『ska-Jodko K. Clinical Outcomes after Uncomplicated Cataract Surgery with Implantation of the Tecnis Toric Intraocular Lens. J Ophthalmol. 2016;2016:3257217.

15. Sheppard AL, Wolffsohn JS, Bhatt U, Hoffmann PC, Scheider A, Hütz WW, et al. Clinical outcomes after implantation of a new hydrophobic acrylic toric IOL during routine cataract surgery. J Cataract Refract Surg. 2013;39:41-7

16. Ferreira TB, Almeida A. Comparison of the visual outcomes and OPDscan results of $A M O$ Tecnis toric and Alcon Acrysof IQ toric intraocular lenses. J Refract Surg. 2012;28(8):551-5.

17. Miyake T, Kamiya K, Amano R, lida Y, Tsunehiro S, Shimizu K. Longterm clinical outcomes of toric intraocular lens implantation in cataract cases with preexisting astigmatism. J Cataract Refract Surg. 2014; 40:1654-60.

18. Krall E, Arlt E, Hohensinn M, Moussa S, Jell G, Alió JL, et al. Vector analysis of astigmatism correction after toric intraocular lens implantation. J Cataract Refract Surg. 2015;41:790-99. 